\title{
Detection Method of High-Speed Low-Altitude Targets for "Luminal" Radar Complex on the Basis of Land-Over-the-Horizon Radars
}

\author{
Valeriy V. Zamaraeva, \\ Aleksandr E. Chentsov and Igor V. Lyutikov*c \\ ${ }^{a} A S D$ troops Central Research Institute \\ of the Russian Federation Defense Ministry \\ 32 Quay Afanasiy Nikitin, Tver, 170026, Russia \\ ${ }^{b}$ ASD MA named Marshal of the Soviet Union Georgiy Zhukov \\ 50 Zhigareva Str., Tver, 170022, Russia \\ 'Siberian Federal University \\ 79 Svobodny, Krasnoyarsk, 660041, Russia
}

Received 07.02.2017, received in revised form 17.03.2017, accepted 27.04.2017

This article analyzes the possibilities of the existing system of air intelligence of the enemy in connections and associations of ASF (ASD). Designated problem that when applying the cruise missiles significantly increased the possibility of enemy aircraft on drawing the sudden impact on objects of strategic nuclear forces and the highest levels of management. Showing alternatives to enemy air reconnaissance systems, to increase the possibility of detection of cruise missiles existing information by means of a system of ASD. Proposed improved detection method based on spectral analysis of signals received from objectives through radar and rear-illuminated tracing enables you to detect and classify targets like cruise missiles. Graphic dependences are analysed accuracy characteristics of measured parameters taken by the echo-signals from RADAR parameters on the basis of the proposed method, appreciated its advantages and disadvantages in contrast to the known (existing) provides conclusions and recommendations for use.

Keywords: detection, air opponent, cruise missiles, over-the-horizon RADAR, ASD, ASF, spectral analysis, transmissive radiolocation, classification purposes.

Citation: Zamaraev V.V., Chentsov A.E., Lyutikov I.V. Detection method of high-speed low-altitude targets for «luminal» radar complex on the basis of land-over-the-horizon radars, J. Sib. Fed. Univ. Eng. technol., 2017, 10(4), 484-496. DOI: 10.17516/1999494X-2017-10-4-484-496.

(C) Siberian Federal University. All rights reserved

* Corresponding author E-mail address: ppzama@mail.ru, lyutikovigor@mail.ru 


\title{
Метод обнаружения скоростных маловысотных целей
}

\section{для «просветного» радиолокационного комплекса \\ на базе загоризонтных РЛС наземного базирования}

\author{
В.В. Замараев ${ }^{\mathrm{a}}$, А.Е. Ченцов ${ }^{\sigma}$, И.В. Лютиков ${ }^{\mathrm{B}}$

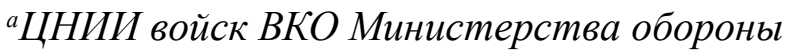 \\ Российской Федерачии \\ Россия, 170026, Тверь, ул. Набережная Афанасия Никитина, 32 \\ ${ }^{6}$ ВА ВКО имени Маршала Советского Союза Г.К. Жукова \\ Россия, 170022, Тверь, ул. Жигарева, 50 \\ ${ }^{6}$ Сибирский федеральный университет \\ Россия, 660041, Красноярск, пр. Свободный, 79
}

\begin{abstract}
В статье проведен анализ возможностей существующей системы разведки воздушного противника в соединениях и объединениях ВКС (ПВО-ПРО). Обозначена проблема, что при применении крылатых ракет существенно увеличены возможности воздушного противника по нанесению внезапного удара по объектам стратегических ядерных сил и высших звеньев управления. Показаны альтернативные варианты построения систем разведки воздушного противника, позволяющие увеличить возможности обнаружения крылатых ракет существующими информационными средствами системы ВКО. Предложен усовершенствованный метод обнаружения, основанный на спектральном анализе сигналов, полученных от иелей с помощью просветной радиолокащии, и позволяющий обнаруживать и классифицировать иели как крылатые ракеты. Проанализированы графические зависимости точностных характеристик измеряемых параметров принимаемых эхосигналов от параметров РЛС на основе предложенного метода, оченены его преимущества и недостатки в отличие от известных (существующих), приведены выводы и рекомендации к применению.
\end{abstract}

Ключевые слова: обнаружение, воздушный противник, крылатые ракеты, ЗГО, загоризонтная РЛС, ВКО, ВКС, спектральный анализ, просветная радиолокация, классификация цели.

Особую опасность при нанесении превентивного разоружающего удара по группировкам стратегических ядерных сил (СЯС), состоящим из мобильных и защищенных стационарных ракетных комплексов, сегодня представляют имеющиеся на вооружении США и стран НАТО крылатые ракеты воздушного и морского базирования (КР). Это объясняется их способностью осуществлять полет на большие дальности на предельно малых высотах с огибанием рельефа местности, возможностью обхода информационных и огневых зон системы ВКО и малой радиолокационной заметностью КР.

Анализ возможностей существующей системы разведки воздушного противника в соединениях и объединениях ВКС (ПВО-ПРО) показал, что при применении КР существенно увеличиваются возможности воздушного противника по нанесению внезапного удара по объектам СЯС и высших звеньев управления (ВЗУ), причем удара, практически не контролируемого существующими средствами обнаружения ВКС. Эти средства в основном представлены средствами радиолокационной разведки, а процесс добывания сведений о КР выполняется дежурными силами и средствами радиотехнических полков, РЛС Федеральной аэронавигационной 
службы и ограниченно - радиолокационными станциями автономных средств целеуказания зенитных ракетных полков [1-3].

Как показал анализ, проведенный в [4], пространственные возможности радиолокационной системы ВКС на малых и предельно малых высотах ограничены и обеспечивают контроль воздушного пространства до 18-23 \% государственной границы РФ. Зоны разведки и контроля воздушного пространства на средних и малых высотах на Северо-востоке страны, на Северном Урале, а также в районах Восточной и Западной Сибири имеют очаговый характер, а в районах Крайнего Севера практически отсутствуют $[4,5]$. Создание сплошного радиолокационного поля на малых высотах над всей территорией страны по экономическим ограничениям не реализуемо. Это обстоятельство приводит к необходимости поиска альтернативных вариантов, позволяющих увеличить возможности обнаружения крылатых ракет существующими информационными средствами системы ВКО.

В настоящее время предлагается несколько таких вариантов.

Первый вариант - использование РЛС на воздушных носителях (аэростатах, вертолетах), что позволяет обнаруживать КР на достаточно больших дальностях. Основной недостаток большая стоимость эксплуатации, что ограничивает возможности их применения при непрерывном дежурстве. Кроме того, возможности по непрерывному применению РЛС на аэростатах существенно ограничиваются метеоусловиями.

Второй вариант предполагает использование маловысотных РЛС, размещенных на штатных или специальных вышках. Имеется несколько типов таких РЛС и радиолокационных комплексов (РЛК) (например, «Каста 2-2», «Подлет», «Снаряд-У» («Барьер») и др.). Возможности РЛС позволяют осуществлять непрерывное боевое дежурство, но имеют недостатки - незначительное увеличение зон обнаружения РЛС на предельно малых высотах и их «стационарность», определяемая требованиями, заданными параметрами радиолокационного поля вокруг прикрываемых объектов, что позволяет противнику на этапе планирования удара произвести разведку и при необходимости организовать помеховое и (или) огневое подавление таких РЛС. Кроме того, иметь мобильный резерв на базе указанных РЛС для прикрытия важных объектов весьма затруднительно.

Третий вариант - применение РЛС загоризонтного обнаружения (РЛС ЗГО), которые позволяют контролировать большие и достаточно удаленные районы при любых высотах полета целей, могут находиться на значительном расстоянии от предполагаемых маршрутов полета КР. Однако существует проблема в применении РЛС ЗГО - это недостаточный потенциал для обнаружения крылатых ракет, который составляет около 15-20 дБ/Вт.

Принципиальной особенностью существующих радиолокационных станций, в том числе приведенных выше, является использование традиционных методов получения радиолокационной информации о воздушных целях. Так, дальность определяется временем запаздывания отраженного от цели сигнала, а азимут - формированием диаграмм антенны в горизонтальной плоскости.

Для решения проблемы, связанной с применением РЛС ЗГО для обнаружения маловысотных целей (в том числе одиночных), предлагается метод обнаружения, основанный на спектральном анализе сигналов, полученных от целей с помощью просветной локации, и позволяющий обнаруживать и классифицировать цели как КР. Этот метод предполагает соз- 
дание «просветного» РЛК на базе передающей позиции РЛС ЗГО и двух приемных позиций, расположенных в районе, в котором ожидается пролет КР. Важной особенностью расположения позиций является ортогональность их базы линии визирования «передатчик - приемник». Прием сигналов осуществляется на слабонаправленную антенну (типа полуволновый вибратор).

При разработке облика такого комплекса должны быть определены параметры комплекса, которые обеспечивают требуемые характеристики обнаружения и классификации целей, и принципы обработки информации для принятия решения о принадлежности обнаруженных целей к классу «КР».

Для решения поставленных задач необходимо развитие теории применительно к рассматриваемой структуре комплекса (в первую очередь выбор обнаружителя и измерителей параметров сигналов).

Энергетический выигрыш метода можно определить соотношением параметров существующей РЛС ЗГО к параметрам предлагаемого комплекса:

- соотношением дальностей обнаружения $v_{\mathrm{r}}=\frac{\mathrm{R}_{\text {пер }}^{2}}{\mathrm{R}_{\text {пр }}^{2}}$,

- соотношением ЭПР цели при просветной и обычной локации $v_{\ni}=\frac{\sigma_{\text {об }}}{\sigma_{\text {прос }}}$,

- соотношением площадей приемных антенн $v_{\mathrm{a}}=\frac{\mathrm{S}_{\text {пр зго }}}{\mathrm{S}_{\text {пр о }}}$,

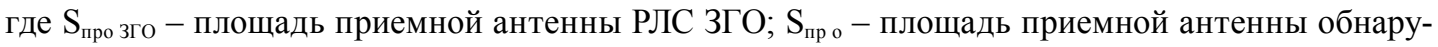
жителя приемника,

- соотношением за счет увеличения времени когерентного накопления (в том числе за счет использования дополнительно внешней когерентности) $v_{\text {нак }}=\sqrt{\frac{\tau_{\text {кн.прос }}}{\tau_{\text {кноб }}}}$.

Суммарный выигрыш для комплекса $v_{\Sigma}=v_{\mathrm{r}} \nu_{9} v_{\mathrm{a}} v_{\text {нак }}$.

Все перечисленные выше коэффициенты могут быть рассчитаны аналитически, кроме соотношения ЭПР при просветной и обычной локации $\left(v_{э}\right)$. В литературе [6-8] считается, что это соотношение может достигать значений 25-42 дБ, а определение конкретного значения требует проведения экспериментальных работ.

Для примера возьмем $v_{\text {нак }}=4 \cdot 10^{4}, v_{\text {нак }}=6, v_{\mathrm{a}}=10^{-3}$, тогда $v_{\Sigma}=240 v_{\text {э. }}$.

Приведенный пример иллюстрирует наличие потенциальных запасов по энергетике, которое позволяет обнаружить одиночные КР и решить проблему с недостающим потенциалом.

Таким образом, если разместить приемные позиции в «ракетоопасном» районе так, чтобы в пределах прямой видимости выполнялись условия обнаружения КР «на просвет» с использованием сигнала от действующих РЛС ЗГО, а при обработке сигналов, обнаруженных на приемных позициях, использовать режим внешней когерентности и согласованную фильтрацию, например, ЛЧМ-сигналов (типа «режим синтезированной апертуры»), то на приемных позициях могут быть реализованы дальности обнаружения малоразмерных, низколетящих целей в диапазоне 15-20 км, что близко к потенциально возможным дальностям обнаружения целей

$$
-487-
$$




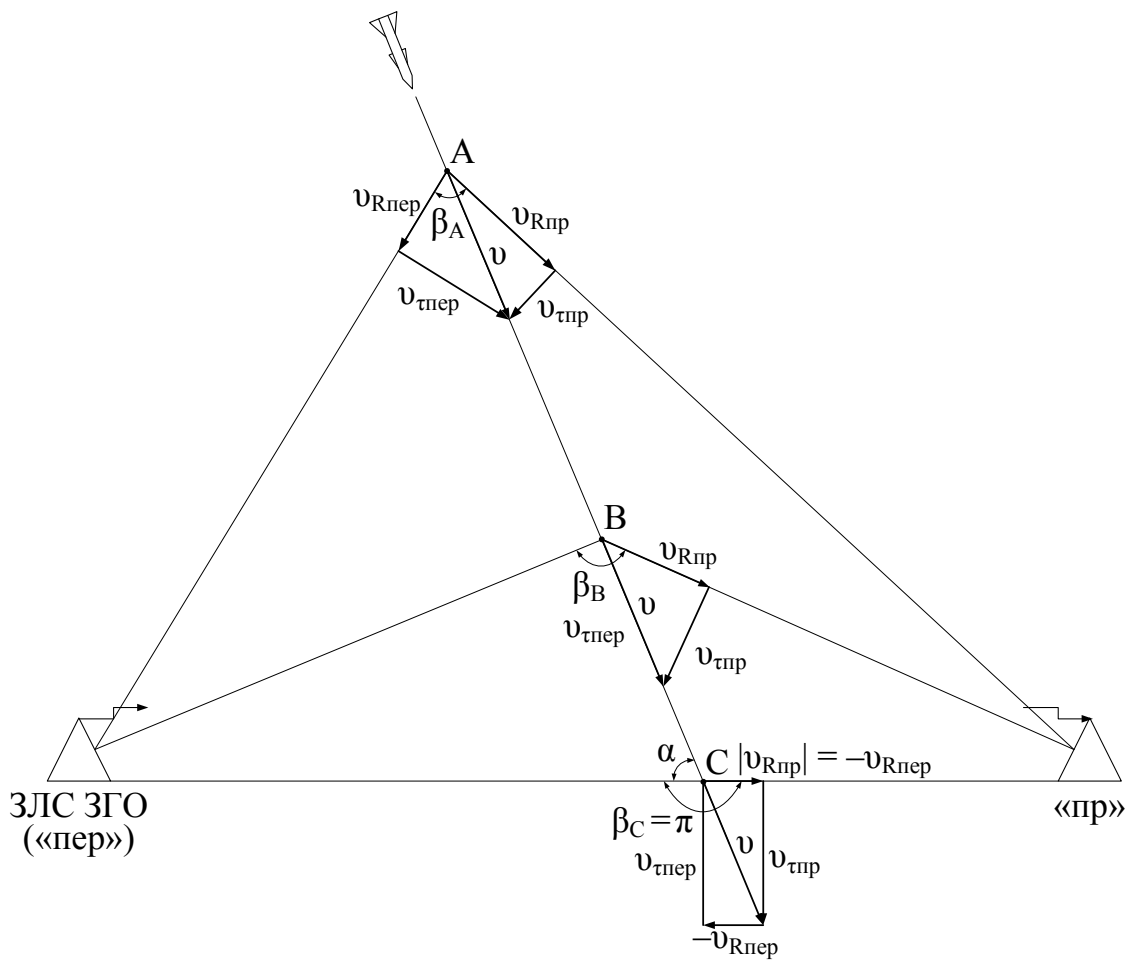

Рис. 1. Геометрическая интерпретация метода на примере одной приемной позиции

на предельно малых высотах по энергетическим ограничениям. Необходимо отметить, что существующие ограничения РЛС ЗГО по времени функционирования (из-за геофизических условий) позволяют использовать ее только в качестве дополнительного средства в системе предупреждения об ударах КР.

Рассмотрим геометрическую интерпретацию метода в случае, когда имеется одна приемная позиция (рис. 1).

Пусть траектория полета цели пересекает линию визирования под углом $\alpha$ в точке $\mathrm{C}$ и проходит через точки А, В, и С. В точке А зондирующий по отношению к цели и отраженный по отношению к приемной станции сигналы имеют радиальную и тангенциальную составляющие вектора скорости цели. В точке В зондирующий сигнал имеет только тангенциальную составляющую, совпадающую с вектором скорости цели, а отраженный сигнал - полный вектор скорости. Точка $\mathrm{C}$ находится на линии визирования «передатчик - приемник», в этой точке принимаемый целью и отраженный от нее сигнал имеет ряд свойств: во-первых, радиальные скорости зондирующего сигнала «передатчик - цель» и отраженного сигнала «цель - приемник» имеют противоположные знаки $\left(v_{\mathrm{R} \text { пер }}=-v_{\mathrm{R}}\right)$, следовательно, доплеровское смещение частоты сигнала от цели на приемной позиции будет равно нулю; во-вторых, вектор скорости цели будет иметь тангенциальные составляющие (по отношению и к передатчику, и к приемнику), а их значения будут определяться углом $\alpha$; в-третьих, бистатический угол «передатчик - цель - приемник» будет составлять $\beta=180^{\circ}$, что соответствует максимальному значению бистатической ЭПР цели. 
Наличие тангенциальной составляющей скорости цели $v_{\tau}=v \times \sin \alpha$ приводит к частотной модуляции принимаемых сигналов. При этом их частотная девиация определяется из выражения

$$
\frac{\mathrm{df}}{\mathrm{dt}}=\frac{\mathrm{v}_{\tau}^{2}}{\mathrm{R}_{\text {пер }} \lambda}+\frac{\mathrm{v}_{\tau}^{2}}{\mathrm{R}_{\text {пр }} \lambda} .
$$

Так как $\mathrm{R}_{\text {пер}}>>\mathrm{R}_{\text {пр }}$, то зависимостью частотной девиации от дальности «передатчик - цель» $\left(\mathrm{R}_{\text {пер }}\right)$ можно пренебречь. Тогда за время когерентного накопления сигнала $\left(\tau_{\text {кн }}\right)$ девиация частоты:

$$
\Delta \mathrm{f}=\tau_{\mathrm{KH}} \frac{\mathrm{df}}{\mathrm{dt}} .
$$

Согласованный фильтр для такого сигнала - ЛЧМ-фильтр с нулевой начальной частотой и указанной девиацией. Полоса фильтра будет определяться $\tau_{\text {кн }}$, девиация будет зависеть от двух параметров $v_{\tau}, \mathrm{R}_{\text {пр }}$, что вызывает неопределенность в оценках $v_{\tau}$ и $\mathrm{R}_{\text {пр }}$ (если дальность $\mathrm{R}_{\text {пр }}$ не измеряется).

Для обнаружения целей с неизвестной частотной девиацией нужно иметь гребенку фильтров, настроенных на разную девиацию частоты.

Отметим, что настроенный на заданный закон изменения доплеровский фильтр позволяет обнаруживать факт пересечения линии «передающая позиция - приемная позиция», но не дает возможноость определить направление, при котором произошло пересечение, так как в выражении (1) используется квадрат тангенциальной скорости цели $\left(v_{\tau}^{2}\right)$.

Для определения направления траектории цели, пересекающей линию визирования «передатчик - приемник», необходимо иметь еще одну позицию. При этом проход цели через две линии визирования будет осуществляться последовательно. Кроме того, в моменты, когда цель не находится на линии визирования, у нее появляется сигнал, доплеровская частота которого не равна нулю, меняется и закон изменения ЛЧМ-сигнала.

Для маловысотных целей предельная дальность обнаружения определяется известным выражением

$$
\mathrm{R}=4,12(\sqrt{\mathrm{h}}+\sqrt{\mathrm{H}})
$$

Расстояние до таких целей будет небольшим. Условие появления ЛЧМ-сигналов от целей

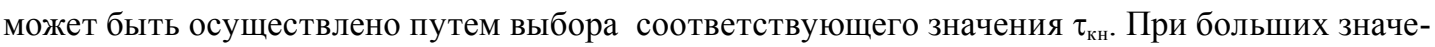
ниях требуемого времени когерентного накопления целесообразно применить метод внешней когерентности, используя при фильтрации в качестве опорного сигнала прямой сигнал передатчика. При этом будут компенсированы спектральные искажения на трассе распространения «передатчик - цель - приемник».

Рассмотрим геометрическую интерпретацию метода, когда имеются две приемные позиции (Пр $\mathrm{A}_{1}$ и Пр $\mathrm{A}_{0}$ ) (рис. 2), расположенные на расстоянии $\mathrm{R}_{\sigma}$ друг относительно друга и ортогонально к линии визирования (передатчик - приемник).

Пусть цель движется под углом $\alpha$ к линии визирования, ее траектория пересекает линию визирования (Пер $\mathrm{A}-$ Пр $\mathrm{A}_{1}$ ) в точке $\mathrm{B}_{1}$, удаленной от Пр $\mathrm{A}_{1}$ на расстояние $\mathrm{R}_{11}$. Скорость цели равна $v$.

$$
-489-
$$




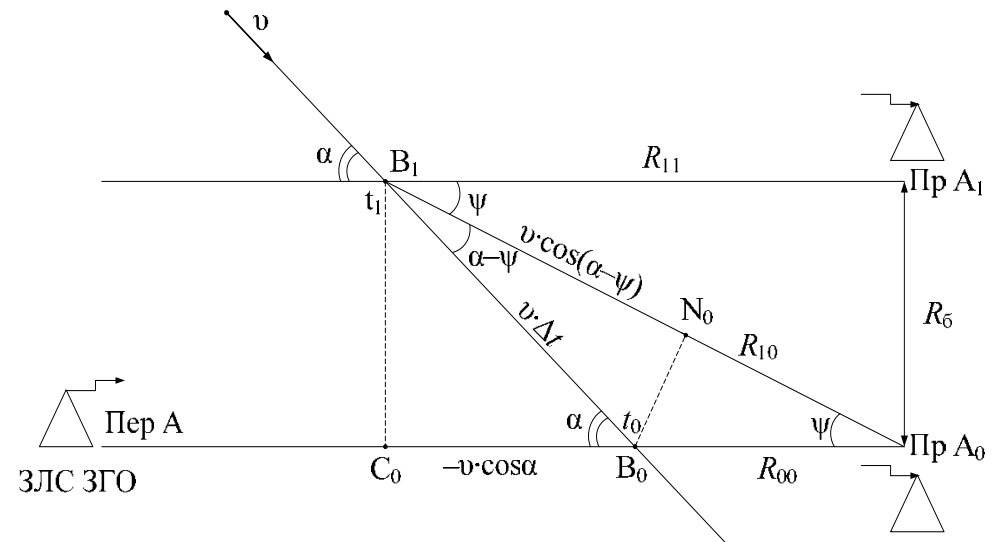

Рис. 2. Геометрическая интерпретация метода на примере двух приемных позициях

На первой приемной позиции, как было отмечено выше, доплеровское смещение сигнала от цели в точке $\mathrm{B}_{1}$ равно 0 , а частотная модуляция сигнала за счет тангенциальной составляющей определяется выражением

$$
\frac{\mathrm{df}}{\mathrm{dt}}=\frac{v_{\tau}^{2}}{\mathrm{R}_{11} \lambda}=\frac{\mathrm{v}^{2} \sin \alpha}{\mathrm{R}_{11} \lambda} .
$$

Рассмотрим формульные зависимости для определения доплеровского смещения и частотной девиации на позициях Пр $\mathrm{A}_{1}$ и Пр $\mathrm{A}_{0}$ в момент времени $\mathrm{t}_{1}$, когда цель находится в точке $\mathrm{B}_{1}$ :

1. $\mathrm{f}_{\text {д1 }}\left(\mathrm{t}_{1}\right)=0, \mathrm{f}_{1}^{\prime}\left(\mathrm{t}_{1}\right)$ - измерения на первой позиции, когда цель находится в точке $\mathrm{B}_{1}$

$$
\mathrm{f}_{1}^{\prime}=\frac{\mathrm{v}^{2} \sin \alpha}{\mathrm{R}_{11} \lambda} .
$$

2. $\mathrm{f}_{\text {д0}}\left(\mathrm{t}_{1}\right), \mathrm{f}_{0}^{\prime}\left(\mathrm{t}_{1}\right)$ - измерения на тот же момент времени на позиции Пр $\mathrm{A}_{0}$

$$
\begin{aligned}
& \mathrm{f}_{\text {д }}\left(\mathrm{t}_{1}\right)=\frac{\mathrm{v} \cdot \cos (\alpha-\psi)-\mathrm{v} \cdot \cos \alpha}{\lambda} \\
& \mathrm{f}_{0}^{\prime}\left(\mathrm{t}_{1}\right)=\frac{(\mathrm{v} \cdot \sin (\alpha-\psi))^{2}}{\mathrm{R}_{10} \lambda}=\frac{(\mathrm{v} \cdot \sin (\alpha-\psi))^{2}}{\lambda \sqrt{\mathrm{R}_{\sigma}^{2}+\mathrm{R}_{11}^{2}}} \\
& \mathrm{R}_{\sigma}^{2}+\mathrm{R}_{11}^{2}=\left(\frac{\mathrm{R}_{\sigma}}{\sin \psi}\right)^{2}
\end{aligned}
$$

Мы получили четыре уравнения с четырьмя неизвестными $-v, \alpha, \psi, \mathrm{R}_{11}$. Решение системы уравнений можно получить известными численными методами.

Таким образом, при пересечении в точке $\mathrm{B}_{1}$ целью линии визирования можно оценить все параметры движения цели, а имея вторую приемную позицию, оценить и направление ее движения.

Как следует из приведенных выше формул, в общем случае принимаемый на приемных позициях сигнал включает в себя два закона изменения фазы (линейный, вызванный радиальным 
перемещением цели, и квадратичный, вызванный тангенциальной составляющей скорости). Для согласованной фильтрации таких сигналов в общем случае необходима система ЛЧМфильтров в двумерном пространстве $\left(\mathrm{f}_{д}, \mathrm{df} / \mathrm{dt}\right)$. Поиск оптимального фильтра, соответствующего принимаемому сигналу, достаточно сложная процедура, поэтому задачу можно привести к поиску оптимального фильтра в одномерном пространстве $\left(\mathrm{f}_{\mathrm{z}}=0, \mathrm{df} / \mathrm{dt}\right)$.

Это условие выполняется в тот момент, когда цель находится на линии визирования в точке $\mathrm{B}_{1}\left(\right.$ Пер $\mathrm{A}-$ Пр $\left.\mathrm{A}_{1}\right)$ либо в точке $\mathrm{B}_{0}\left(\right.$ Пер $\mathrm{A}-\Pi$ - $\left.\mathrm{A}_{0}\right)$. В этом случае $\mathrm{f}_{\text {д1 }}\left(\mathrm{t}_{1}\right)=0, \mathrm{f}_{\text {д0 }}\left(\mathrm{t}_{0}\right)=0$, а частотная девиация зависит от двух параметров цели - тангенциальной составляющей скорости цели $\left(v_{\tau}\right)$ и дальности до нее $\mathrm{R}_{11}, \mathrm{R}_{00}$. Дополнительный замер на второй линии визирования позволит оценить положение цели, используя только линейки фильтров на каждой позиции (поиск в одномерном пространстве). Если использовать дополнительные замеры (например, от второй позиции, когда на первой позиции $\mathrm{f}_{0}=0$ ), то будем иметь избыточную информацию, которая может быть применена для повышения точности оценки вектора состояния цели.

При двух позициях и расстоянии между ними $\mathrm{R}_{6}$ тангенциальная составляющая скорости

$$
v_{\tau}=\frac{R_{\sigma}}{t_{0}-t_{1}}
$$

где $\mathrm{t}_{0}$ - оценка момента времени пересечения целью линии визирования Пер $\mathrm{A}$ - Пр $\mathrm{A}_{0}$ (по результатам обнаружения сигнала в соответствующем доплеровском фильтре); $\mathrm{t}_{1}$ - оценка момента времени пересечения целью линии визирования Пер А - Пр $\mathrm{A}_{1}$ (по результатам обнаружения сигнала в соответствующем доплеровском фильтре).

Дальность до цели от приемной позиции Пр $\mathrm{A}_{0}$ в момент пересечения соответствующей линии визирования

$$
\mathrm{R}_{0}=\frac{\mathrm{v}_{\tau}^{2}}{\lambda\left(\frac{\mathrm{df}}{\mathrm{dt}}\right)_{0}^{*},}
$$

где $\left(\frac{\mathrm{df}}{\mathrm{dt}}\right)_{0}^{*}$ - оценка частотной девиации сигнала (на основании параметров фильтра, в котором произошло обнаружение сигнала при $\mathrm{f}_{\mathrm{z}}=0$ ).

Радиальная составляющая скорости определяется соотношением

$$
\mathrm{v}_{\tau}=\frac{\mathrm{R}_{0}-\mathrm{R}_{1}}{\mathrm{t}_{0}-\mathrm{t}_{1}}
$$

Рассмотрим более детально вопрос о параметрах фильтра, которые используются для оценки дальности до цели. Как было отмечено, при пересечении целью линии визирования доплеровское смещение сигнала от цели равно нулю (падающая и отраженная волны имеют одинаковый по величине и противоположный по знаку набег фазы), а частотная девиация сигнала от цели определяется выражением (1).

Для согласованной фильтрации такого сигнала надо иметь два фильтра с одинаковой по величине частотной девиации, но с разными начальными частотами Допплера. В связи с тем, что полоса сигнала для рассматриваемого случая включает в себя и область пассивных по- 
мех, необходима режекция сигналов в полосе $\Delta \mathrm{f}_{\mathrm{p}}$ в области $\mathrm{f}_{\mathrm{z}}=0$. Указанные фильтры будут собирать сигналы в полосе $\Delta \mathrm{f}_{\text {д1 }}$ и $\Delta \mathrm{f}_{\text {д2 }}$ соответственно. Сигналы с выхода этих фильтров суммируются когерентно (с учетом фазы, определяемой временным сдвигом сигналов). Решение о пересечении линии визирования принимается по максимальному значению сигнала в двумерной области (время - частотная девиация). Эту процедуру можно реализовать с помощью дискриминатора, на вход которого подаются сигналы с двух указанных выше фильтров.

Рассмотрим ошибки измерения дальности предлагаемого комплекса до цели.

При наличии частотной девиации принимаемых сигналов дальность до цели может быть определена с помощью выражений (9) и (10).

Дисперсию ошибок измерения дальности до цели можно определить методом линеаризации:

$$
\sigma_{\mathrm{R}}^{2}=\left(\frac{2 \mathrm{v}_{\tau}}{\mathrm{f}^{\prime}}\right) \sigma_{\mathrm{v}_{\tau}}^{2}+\left(\frac{\mathrm{v}_{\tau}^{2}}{\left(\mathrm{f}^{\prime}\right)^{2}}\right) \sigma_{\mathrm{f}^{\prime}}^{2}
$$

При этом

$$
\begin{aligned}
& \sigma_{\mathrm{f}^{\prime}}^{2}=\frac{\mathrm{k}}{\mathrm{q}^{2} \tau_{\mathrm{\kappa н}}^{2}}, \quad \sigma_{v_{\tau}}^{2}=\frac{2 v_{\tau}^{2}}{\mathrm{R}_{\sigma}} \sigma_{\mathrm{t}_{\text {пер }}}^{2}, \\
& \mathrm{f}^{\prime}=\frac{\mathrm{df}}{\mathrm{dt}}=\frac{\mathrm{v}^{2} \sin \alpha}{\mathrm{R}_{\text {пр }} \lambda}, \\
& \sigma_{\mathrm{t}_{\text {пер }}}^{2}=\frac{1}{\mathrm{q}^{2} \Pi_{э \phi}^{2}}=\frac{1}{\mathrm{q}^{2} \tau^{2} \mathrm{f}^{\prime}},
\end{aligned}
$$

где $\mathrm{q}$ - отношение сигнал/шум; $\sigma_{\mathrm{t}_{\text {пер }}^{2}}$ - дисперсия оценки момента пересечения целью линии визирования. Для ее вычисления требуется задаться отношением сигнал/шум (для построения зависимостей, приведенных на рис. 3 , принято $\mathrm{q}^{2}=100$ ).

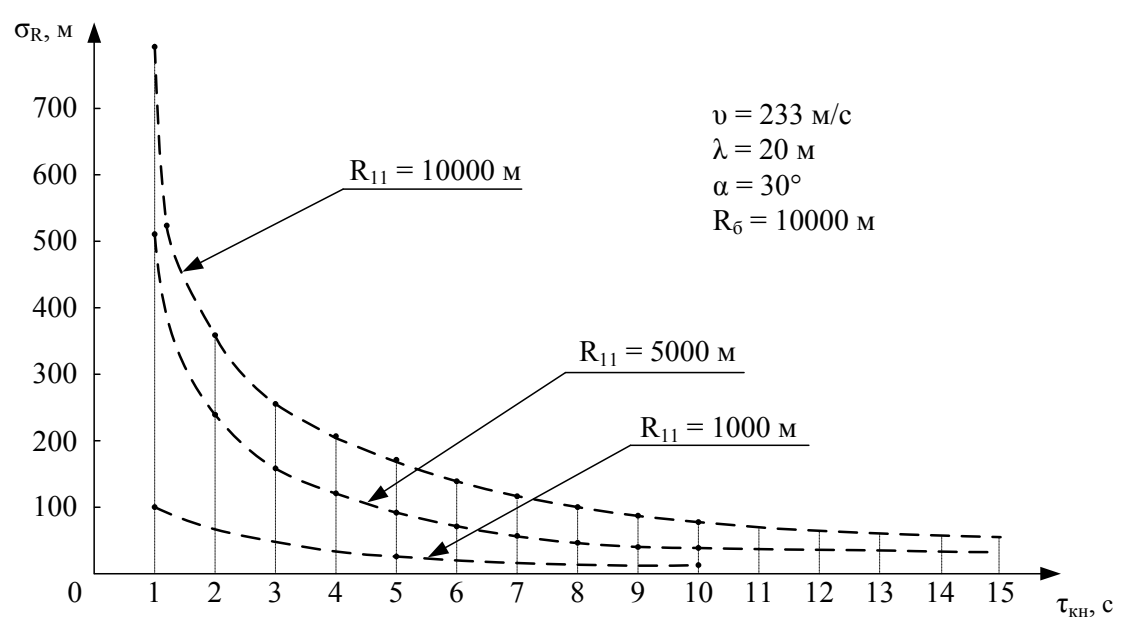

Рис. 3. График зависимостей ошибки измерения по дальности от времени когерентного накопления $\left(\tau_{\text {кн }}\right)$ при различных значениях расстояния от точки пересечения целью линии визирования до приемника первой позиции $\left(\mathrm{R}_{11}\right)$ 
Расчетные результаты, полученные в среде математического моделирования Matlab, представлены на рис. 3-6.

Из данных рисунка 3 следует, что даже при времени когерентного накопления от 3 до 5 с параметры ошибок измерения дальности исследуемого комплекса до цели на два-три порядка меньше по сравнению с ошибками измерения по дальности РЛС ЗГО. При времени когерентного накопления более 10 с ошибки измерения по дальности практически не меняются.

На рис. 4 приведены графики зависимости ошибок определения дальности до цели от расстояния между приемными станциями $\left(\mathrm{R}_{6}\right)$ при времени когерентного накопления $\left(\tau_{\text {кн }}\right) 5$ и $10 \mathrm{c}$. По данным графиков можно заключить, что при $\mathrm{R}_{6} \geq 10000$ м ошибки измерения по дальности практически постоянны.

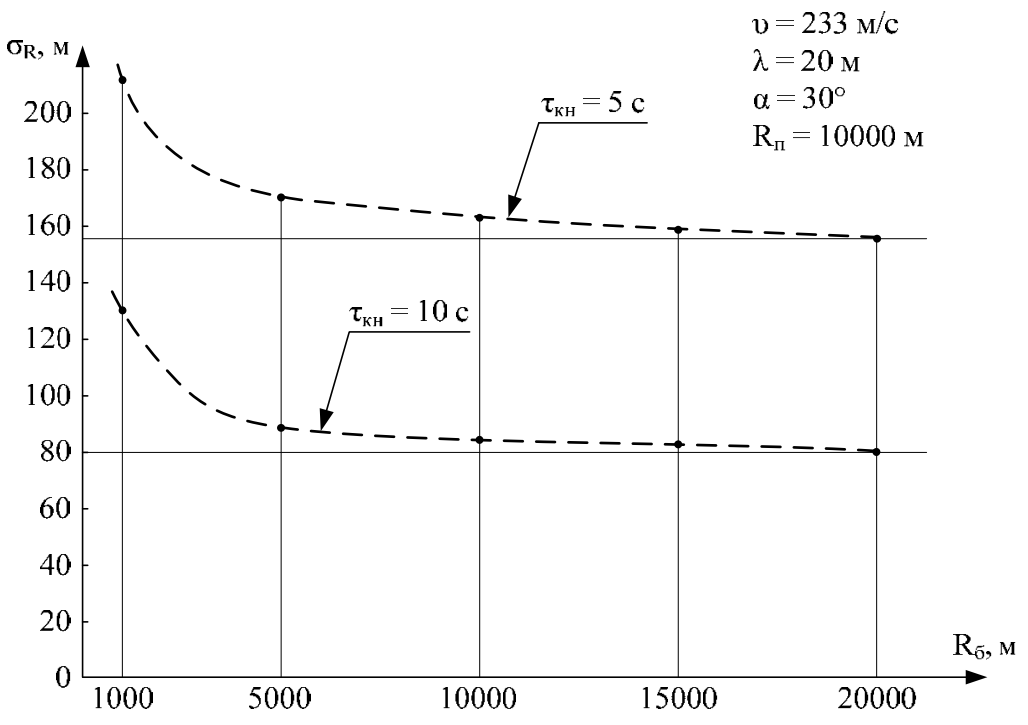

Рис. 4. График зависимости ошибок определения дальности до цели от расстояния между приемными станциями при различных значениях времени когерентного накопления

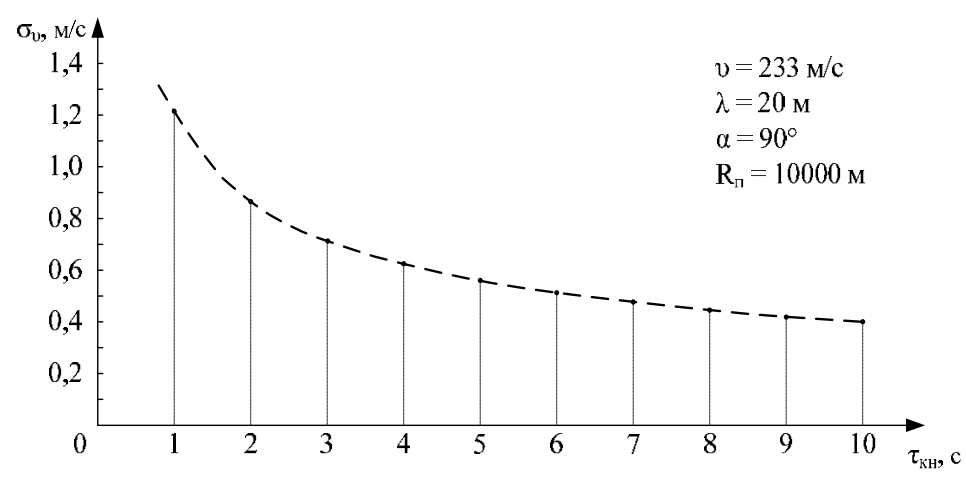

Рис. 5. График зависимости ошибки измерения путевой скорости цели от времени когерентного накопления 
На рис. 5 представлен график зависимости ошибки измерения путевой скорости цели (для простоты расчетов было принято $\alpha=90^{0}$, т.е. тангенциальная составляющая вектора скорости равна полному вектору скорости) от времени когерентного накопления $\left(\tau_{\text {кн }}\right)$.

На графике видим, что при $\tau_{\text {кн }}=10$ с ошибка измерения полного вектора скорости $\left(\sigma_{v}\right)$ равна примерно 0,45 м/с. Полученная оценка ошибки измерения путевой скорости исследуемого комплекса на два порядки меньше ошибки определения путевой скорости РЛС ЗГО, что позволяет достаточно точно определить скорость полета цели и совместно с признаком малой высоты траектории полета идентифицировать ее как крылатую ракету.

На рис. 6 представлен график зависимости определения ошибок по дальности от длины волны передатчика, из которого следует, что при увеличении длинны волны характеристики обнаружителя значительно улучшаются.

Признак того, что цель летит на малой высоте, может быть вычислен косвенно по длительности получения сигнала от цели (короткая траектория).

Рассмотрим изменение $\mathrm{f}^{\prime}$ в зависимости от расстояния до цели (при пересечении линии визирования) для различных длин волн.

Частотная девиация сигнала за время когерентного накопления определяется выражением $\Delta \mathrm{F}=\mathrm{f}^{\prime} \tau_{\mathrm{kн}}$.

В зависимости от соотношения $\Delta \mathrm{F}$ и $\Delta \mathrm{f}_{д}=\frac{1}{\tau_{\text {кн }}}$

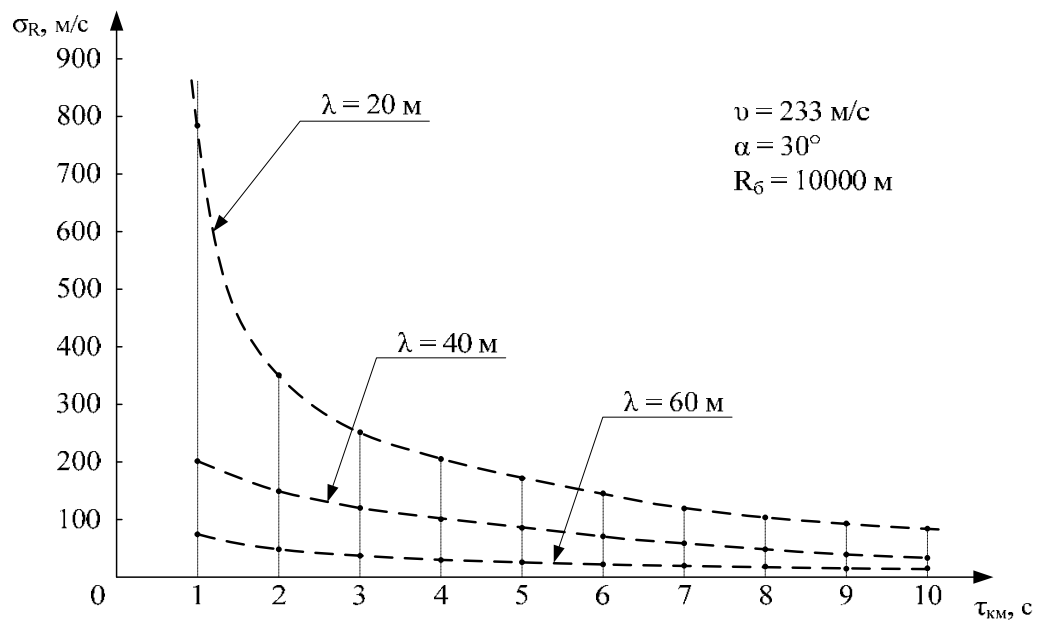

Рис. 6. График зависимости определения ошибок по дальности при различных значениях длины волны передатчика

Таблица 1

\begin{tabular}{|c|c|c|c|c|c|}
\hline$\lambda / \mathrm{R}$ & 20000 & 10000 & 5000 & 2,5000 & 1000 \\
\hline 20 & 0,25 & 0,5 & 1 & 2 & 5 \\
\hline 40 & 0,125 & 0,25 & 0,5 & 1 & 2,5 \\
\hline 60 & 0,063 & 0,125 & 0,25 & 0,5 & 1,25 \\
\hline
\end{tabular}




$$
\mathrm{n}=\frac{\Delta \mathrm{F}}{\Delta \mathrm{f}_{\text {д }}}=\mathrm{f}^{\prime} \tau_{\text {кн }}^{2}
$$

влияние частотной девиации различно. Так, при времени когерентного накопления, равного $1 \mathrm{c}$, влияние девиации проявляется на дальностях менее 5 км в коротковолновой части диапазона. При времени когерентного накопления 10 с влияние существенно на дальностях до 20 км во всем диапазоне частот. Не рассматривая другие факторы, влияющие на эффективность метода, можно в первом приближении считать, что время когерентного накопления в интересах решения задачи обнаружения маловысотных целей должно быть 10 с.

\section{Выводы}

1. Метод предлагается реализовать в виде РЛ-комплекса, использующего передающую позицию ЗГ РЛС и две приемных позиции, расположенных в необходимом для контроля районе ортогонально к линии визирования. Комплекс позволяет надежно обнаруживать крылатые ракеты в прикрываемом районе и с высокой точностью оценивать параметры их движения, необходимые для классификации степени их опасности.

2. Предлагаемый метод в отличие от известных (существующих) использует для измерения дальности до цели спектральные особенности отраженных сигналов.

3. Время когерентного накопления полезного сигнала на обнаружителе комплекса от 3 до 5 с позволяет получить ошибки определения дальности и скорости цели на несколько порядков меньше по сравнению с теми же характеристиками РЛС ЗГО.

4. При выборе расстояния между приемными станциями комплекса более 10 км значение ошибки определения дальности до цели не изменяется.

5. Увеличение длины волны передающей станции в диапазоне от 20 до 60 м значительно (в 2-3 раза) улучшает характеристики обнаружителя.

\section{Список литературы}

[1] Лобов С.А., Ляпоров В.Н. Угрозы национальной безопасности России. Военные угрозы. Тверь: ВА ВКО имени Маршала Советского Союза Г.К. Жукова, 2014. [Lobov S.A, Liaporov V.N. Threats to Russia's national security. Military threats. Tver: ASD MA named Marshal of the Soviet Union Georgiy Zhukov, 2014 (in Russia)]

[2] Бояринцев А, Пашенных В. Перспективы радиолокационной разведки. $B K O, 2006,4$. [Boiarintsev A, Pashennykh V. Prospects for radar reconnaissance. ASD Journal, 2006, 4 (in Russia)]

[3] Милешкевич B.A. ВBC без глаз и ушей. BKO, 2008, 2. [Mileshkevich V.A. The AIR FORCE without the eyes and ears. ASD Journal, 2008, 2 (in Russia)]

[4] Шрамченко А. Радиотехнические войска: трудное настоящее. $B K O, 2005,1$. [Shramchenko A. Radiotechnical troops: hard now. ASD Journal, 2005, 1 (in Russia)]

[5] Кислуха А. К единому радиолокационному полю страны. BKO, 2012, 2. [Kislukha A. A single radar field country. ASD Journal, 2012, 2 (in Russia)]

[6] Глазер Дж.И. Некоторые результаты по определению двухпозиционной ЭПО сложных объектов. Труды Института инженеров по электротехнике и радиоэлектронике (ТИИЭР), 1989, 77(5), 8-18. [Glazer J.I. Some results on the definition of two-step EPO complex objects. Transactions of the Institute of electrical and electronics engineers (TEEE), 1989, 77(5), 8-18 (in Russia)]

$$
-495-
$$


[7] Григас С.Э, Литовченко Д.Ц, Скорынин А.А. Контроль космического пространства с использованием просветной радиолокации. Антенны, 2013, 1, 83-86. [Grigas S.E, Litovchenko D.Ts, Skorynin A.A. Control of outer space using the rear-illuminated tracing, Antennas, 2013, 1, 83-86 (in Russia)]

[8] Ковалев Ф.Н. Автореферат дисс. ... д-ра техн. наук. Нижегородский государственный технический университет имени Р.Е. Алексеева. 2015, 30. [Kovalev F.N. Thesis for the degree of doctor of technical sciences. Nizhny Novgorod State Technical University named R.E. Alexeev. 2015, 30 (in Russia)] 